\title{
Implementations of Signal-Processing Algorithms for OFDM Systems
}

\author{
Ming-Der Shieh, ${ }^{1}$ Yin-Tsung Hwang, ${ }^{2}$ Hanho Lee, ${ }^{3}$ Chirn Chye Boon, ${ }^{4}$ and Zhiyuan Yan ${ }^{5}$ \\ ${ }^{1}$ Department of Electrical Engineering, National Cheng Kung University, Tainan 600, Taiwan \\ ${ }^{2}$ Department of Electrical Engineering, National Chung Hsing University, Taichung 40227, Taiwan \\ ${ }^{3}$ Department of Information and Communication Engineering, Inha University, Inncheon, Republic of Korea \\ ${ }^{4}$ School of Electrical \& Electronic Engineering, Nanyang Technological University, Singapore \\ ${ }^{5}$ Department of Electrical \& Computer Engineering, Lehigh University, Bethlehem, PA 18015, USA \\ Correspondence should be addressed to Ming-Der Shieh, shiehm@mail.ncku.edu.tw
}

Received 31 May 2012; Accepted 31 May 2012

Copyright (C) 2012 Ming-Der Shieh et al. This is an open access article distributed under the Creative Commons Attribution License, which permits unrestricted use, distribution, and reproduction in any medium, provided the original work is properly cited.

Orthogonal frequency-division Multiplexing (OFDM) techniques, coupled with multiple-input multiple-output (MIMO) processing, have been widely deployed in various wireless communication systems such as $802.11 \mathrm{n}$, WiMAX, and 3GPP LTE. To enhance the system performance, novel signal processing techniques are developed, which often require massive and complicated computations and pose major challenges in implementations. With the advances of VLSI technology, the employment of these sophisticated signal processing algorithms now becomes feasible via dedicated mapping onto powerful digital signal processors or dedicated hardware modules. Joint consideration of algorithm development and implementation issues is crucial to achieve optimized solutions. This special issue is created to bring together the state-of-the-art research contributions to the digital signal processing techniques tailored for OFDM systems. It has indeed successfully attracted the submissions of many high-quality papers. After going through a conscientious peer-review process, eight papers are selected. These papers make an inspiring ensemble with a topic spectrum ranging from novel algorithm development, theoretical system characterization, to efficient hard-wired implementations in FPGA/ASIC. Although research topics on signal processing for MIMO-OFDM systems are too broad to be covered in one special issue, we believe that the collected papers dutifully serve as a good starting point. Finally, we would like to thank the authors for their excellent contributions and the cooperation during the editorial process. The competent and timely work by all the reviewers of these papers is also highly appreciated.
For the eight papers included in this special issue, two of them aim at channel estimation and tracking. Another two papers work on the MIMO signal detection schemes. Still another two papers are related to Trellis decoder designs. We also have one paper focusing on the mitigation of $\mathrm{RF}$ impairments and another one provides probabilistic behavior analysis of MIMO channels.

Specifically, in the paper entitled "Probabilistic behavior analysis of MIMO fading channels under geometric mean decomposition," P. H. Kuo and P. A. Ting presents statistical characterization of link gains and channel capacity that can be achieved by using a GMD precoding scheme. Problem formulation, PDF and channel capacity derivation, finitestate Markov chains constructions, and simulation results are provided with satisfactory performance.

In the paper entitled "CP-based SBHT-RLS algorithms for tracking channel estimates in multicarrier modulation systems" by $\mathrm{H}$. Ali, two new algorithms that exploit cyclic prefix for data detection and employ systolic block Householder transformation recursive least squares (SBHT-RLS) algorithms for channel tracking are presented. The new methods outperform existing RLS-based channel tracking schemes. Computational complexity and parallel implementation issues are also addressed. Complete algorithm performance evaluations subject to varying forgetting factor parameter values, constellation size, and word lengths are provided to illustrate the advantages of the proposed work.

The paper is entitled "Advanced receiver design for mitigating multiple RF impairments in OFDM systems algorithms and RF measurements." Kiayani et al. investigate the joint 
impact of frequency-selective I/Q imbalance at both transmitter and receiver together with channel distortions and CFO errors. Two estimation and compensation structures based on different pilot patterns are proposed to separate the individual impairments. Low-complexity time domain estimation and compensation algorithms are next developed to mitigate the problems. Both simulation and measurement results support their performance claims.

There are two papers both working on the topic of MIMO-OFDM signal detection. In the paper entitled "Loopreduction LLL algorithm and architecture for lattice-reductionaided MIMO detection," Liao et al. propose a loop reduction LLL algorithm and its architecture for lattice-reductionaided MIMO detection. A look-ahead check technique is applied to reduce the algorithm complexity while observing the original LLL criterion. Simulation results show that not only the computation complexity but also the computing latency are effectively reduced.

In the paper "Two-dimensional iterative processing for $D A B$ receivers based on trellis-decomposition," W. J. Houtum and F. M. J. Willems investigate trellis decoding and iterative techniques for digital audio broadcasting (DAB). Trellisdecomposition methods allow us to estimate the unknown channel phase, since this phase relates to the subtrellises. Simulations of this iterative approach applied to the TU6 (COST207) channel show an improvement of $2.4 \mathrm{~dB}$ at a Doppler frequency of $10 \mathrm{~Hz}$.

In the paper entitled "A power-efficient soft-output detector for spatial-multiplexing MIMO communications," Wang et al. propose VLSI implementation of a configurable power-efficient MIMO detector that supports $4 \times 4$ spatial multiplexing and modulation from QPSK to 64-QAM. A novel tree search algorithm is also proposed to enable the detector to provide soft outputs and to be implemented in parallel and pipelined hardware architecture.

The paper entitled "Interference cancellation using replica signal for HTRCI-MIMO/OFDM in time-variant large delay spread longer than guard interval" focuses on the time domain ISI compensation through the replica signal insertion with the shifted channel impulse response. System models and complexity analysis are also provided. Exhaustive simulations are conducted to show their performance edge.

In the paper entitled "VLSI architectures for slidingwindow-based space-time turbo trellis code decoders," G. Passas and S. Freear present VLSI architectures of space-time turbo trellis coding decoders as well as of a set of SISO-MAP component channel decoders used in turbo coding based on sliding window. They consider space-time turbo-code receivers based on nonbinary trellises. Finally, measurements of complexity and decoding delay of several sliding window technique-based MAP decoder architectures are developed and compared. 

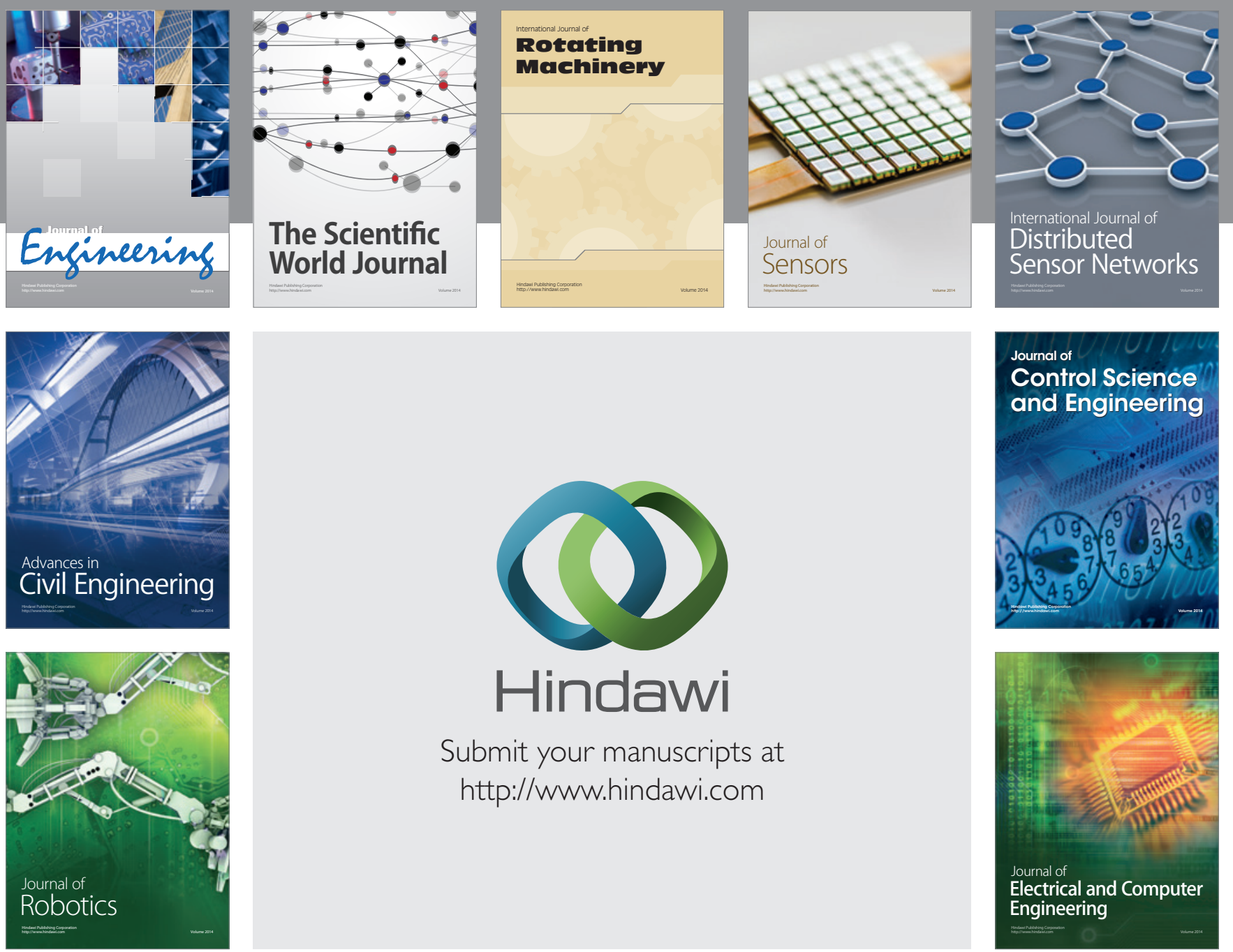

Submit your manuscripts at

http://www.hindawi.com
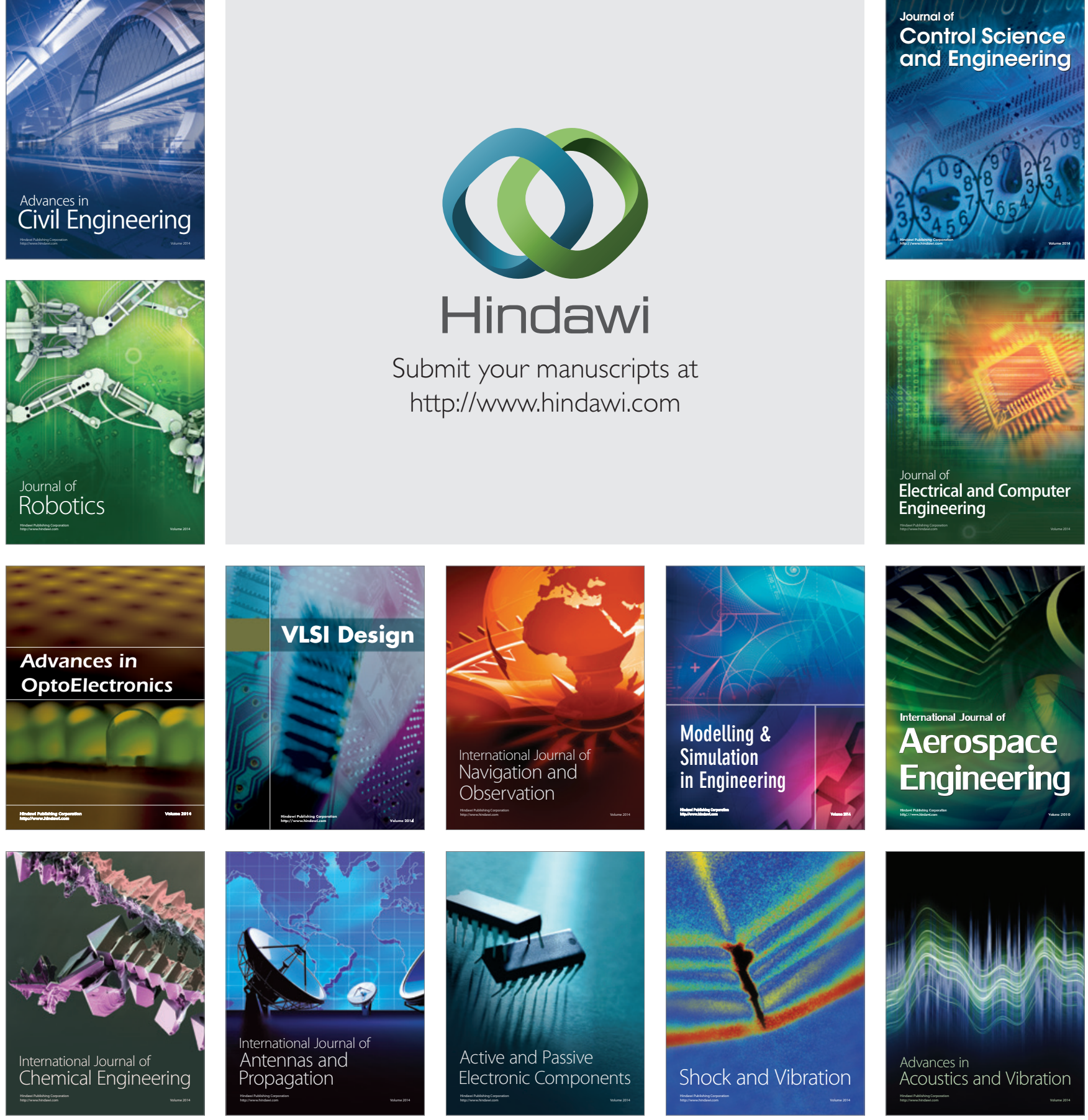